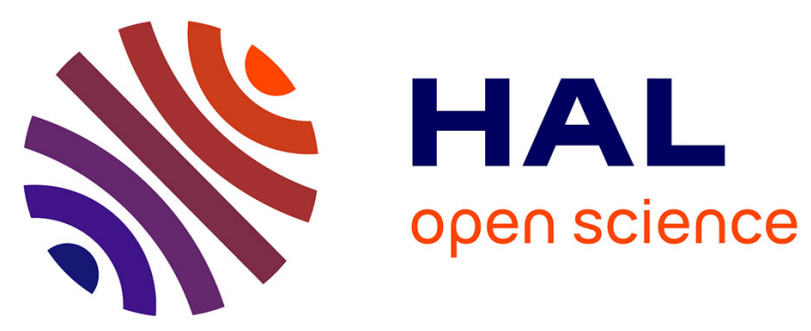

\title{
Consensus-based formation control and obstacle avoidance for nonholonomic multi-robot system
}

Daravuth Koung, Isabelle Fantoni, Olivier Kermorgant, Lamia Belouaer

\section{To cite this version:}

Daravuth Koung, Isabelle Fantoni, Olivier Kermorgant, Lamia Belouaer. Consensus-based formation control and obstacle avoidance for nonholonomic multi-robot system. International Conference on Control, Automation, Robotics and Vision (ICARCV), Dec 2020, Shenzhen, China. 10.1109/ICARCV50220.2020.9305426 . hal-02933732

\section{HAL Id: hal-02933732 \\ https://hal.science/hal-02933732}

Submitted on 8 Sep 2020

HAL is a multi-disciplinary open access archive for the deposit and dissemination of scientific research documents, whether they are published or not. The documents may come from teaching and research institutions in France or abroad, or from public or private research centers.
L'archive ouverte pluridisciplinaire HAL, est destinée au dépôt et à la diffusion de documents scientifiques de niveau recherche, publiés ou non, émanant des établissements d'enseignement et de recherche français ou étrangers, des laboratoires publics ou privés. 


\title{
Consensus-based formation control and obstacle avoidance for nonholonomic multi-robot system
}

\author{
Daravuth Koung, Isabelle Fantoni, Olivier Kermorgant and Lamia Belouaer
}

\begin{abstract}
Managing multiple robots into a formation can be beneficial, especially in logistics sectors where multiple robots can work together to transport larger loads. This paper presents a consensus control law for formation with navigation and obstacle avoidance of multiple wheeled mobile robots. The formation control is based on adapting a consensus algorithm from flocking, and we propose an obstacle avoidance methodology that ensures the formation while navigating around obstacles. Simulations of the control law using four wheeled mobile robots as well as experiments using actual industrial robots are shown in order to validate the theory.
\end{abstract}

\section{INTRODUCTION}

The interest in integrating multi-robot system (MRS) [1] into real-world applications is increasing more and more. In fact, MRS performs better for complex tasks with more efficiency and robustness compared to one single robot [2]. It can be found in a wide range of applications such as in civilian and military sectors including tasks of patrolling [3], exploration and mapping of unknown environments [4]. Logistics sectors can gain great benefits from using MRS as it provides flexibility, reliability as well as extensive loadtransporting capacity in term of weight and size.

Formation control and flocking are the basis of multirobot system, where a team of robots must achieve common desired tasks together. Depending on scenarios, the robot's team formation can be a rigid pattern or flexible. The later behavior is usually inspired by nature, for instance, a flocking of birds. Formation control can be seen as a problem of driving robots to a desire geometric shape, which is, normally, defined by the inter-distance between robots in the network [5]. Flocking, on the other hand, control the group of robots based on three rules [6]: collision avoidance, velocity matching and flock centering. Generally, only kinematic model is involved in the convergence of formation, while dynamic model is usually considered in flocking behavior [7].

Our global research goal is to develop an industrial implementable cooperative load-transporting system, which includes multi-robot task allocations and formation control, using MRS. In this paper, we aim to revise a current promising decentralized flocking algorithm such that it can be used for formation of nonholonomic robots with obstacle

Daravuth Koung is with Laboratoire des Sciences du Numérique de Nantes (LS2N), Ecole Centrale de Nantes (ECN) and E-COBOT, France. daravuth. koungels2n. fr

Isabelle Fantoni is with LS2N and CNRS. Olivier Kermorgan is with LS2N and ECN. isabelle.fantoniels2n.fr, olivier.kermorgant@ec-nantes.fr

Lamia Belouaer is with E-COBOT. 1.belouaerde-cobot. fr avoidance capability, which is crucial for actual implementation of MRS in logistics sectors.

The contributions of this paper are threefold. Firstly, we adapt existing consensus algorithm in [8], which was for double integrator model, to single integrator consensus system. Secondly, we enhance the obstacle avoidance for flocking from [9] such that each robot takes into account its neighbors' obstacles as well, which is suitable for preserving formation. Last but not least, we combine the two algorithms and validate them on wheeled mobile robots in both simulations and real experiments with industrial robots.

The rest of the paper is organized as following. Section II and III present the existing work and the objective of our experiments, respectively. Section IV shows the mathematical theory used in this paper. Our proposed algorithm is introduced in section V. Section VI demonstrates the simulation and experiment results. Finally, conclusion and future work about this paper are given in section VII.

\section{RELATED WORK}

Several strategies for formation control and obstacle avoidance have been proposed in recent years. Consensus algorithm can be seen as one of the most popular control approaches. Generally, consensus is based on graph theory with an objective of reaching an agreement among robots in the network [10]. There exist numerous studies on consensus for second-order system including [11] and [12] while the algorithm for first-order system can be found in [13] and [14]. Leader-follower consensus control laws were proposed by [15] and [16], in which the leader is generally represented by a virtual robot. Despite that, little to no researches have emphasized on validating proposed control laws in actual experiments. In [8], the authors introduced a PID-like decentralized consensus for the double integrator system, which was validated on drones. There are also studies on formation control based on flocking algorithm ([17] and [18]). However, none of them take into account the obstacle avoidance capability. The authors of [9] has introduced an obstacle avoidance approach for flocking by treating each obstacle as a virtual agent. Inspired by this work, the authors of [19] have proposed a flocking and obstacle avoidance algorithm that does not distinguish between a robot and an obstacle, simply said, the controller treats every neighbors as obstacles. In [20] and [21], artificial potential field approach is used for avoiding obstacles in their formation control. Even though there are numerous studies, few of them actually focus on experimental validation. 


\section{PROBLEM STATEMENT}

Let us consider four Huskys [22], $\left(x_{c_{i}}, y_{c_{i}}\right)$ and $\left(x_{c_{g}}, y_{c_{g}}\right)$ are the initial and goal position of their barycenter, respectively. Huskys, which are used for this experiment, are industrial wheeled mobile robots designed for compact and efficient indoor load transportation. Their mission is to navigate cooperatively to any goal point while avoiding obstacle, as shown in Fig. 1. This is one of use cases of formation control in practical implementation, transportation of huge loads in this case. Thus, the following conditions must be satisfied:

- No inter-robot collisions.

- No collision with obstacles.

- They must be able to reach any goal point provided that it is clear from obstacle.

- Formation has to be maintained during the navigation.

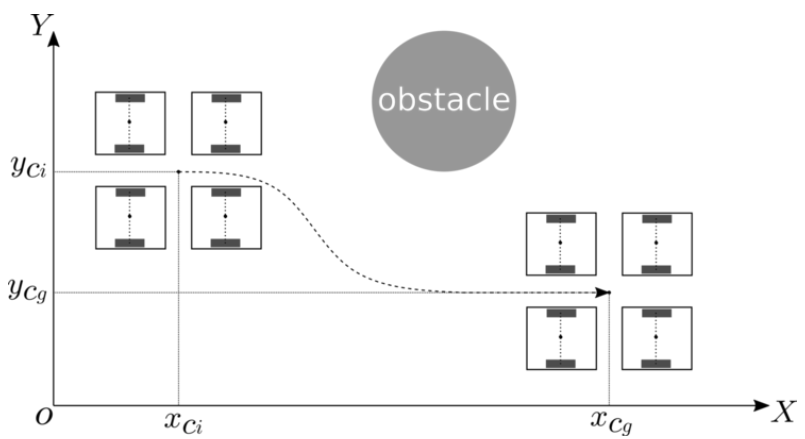

Fig. 1: Formation control and obstacle avoidance.

Furthermore, only local motion control is assumed. Indeed, in a cluttered environment a motion planning phase is necessary before the actual control. The proposed approach, like any state feedback control, would be subject to local minima in many cases if used without a motion planner. However, it can be used as a steering function in classical planning algorithms.

\section{BACKGROUND}

The mathematical and theoretical foundations for experimental work of this paper are based on graph [23] and consensus [24] theory. We focus on the flocking control and the obstacle avoidance algorithm for multi-robot system proposed by [8] and [9], respectively.

\section{A. Graph theory for multi-robot system}

The multi-robot system topology is represented by an undirected graph $G=(\mathcal{V}, \mathcal{E})$ where $\mathcal{V}=\{1,2 \ldots, n\}$ and $\mathcal{E} \subseteq\{(i, j): i, j \in \mathcal{V}, j \neq i\}$ are a set of vertices and edges, respectively. Each robot is denoted by a node of the vertices, and the set of neighbors of node $i$ is defined by:

$$
N_{i}=\{j \in \mathcal{V}:(i, j) \in \mathcal{E}\}=\left\{j \in \mathcal{V}:\left\|\mathbf{p}_{\mathbf{j}}-\mathbf{p}_{\mathbf{i}}\right\|<c\right\}
$$

where $c>0$ is the interaction range between robots, and $\|$.$\| denotes the Euclidean norm. \mathbf{p}_{\mathbf{i}}$ and $\mathbf{p}_{\mathbf{j}}$ are the Cartesian coordinates of robots $i$ and $j$, respectively.
The desired formation of the system can be expressed as:

$$
\left\|\mathbf{p}_{\mathbf{j}}-\mathbf{p}_{\mathbf{i}}\right\|=d \quad \forall j \in N_{i}(\mathbf{p})
$$

where $d$ is the desired inter-distance.

\section{B. Flocking algorithm}

The authors of [8] have proposed a PID-like decentralized control of flocking for a double integrator system whose dynamics are defined as following:

$$
\begin{aligned}
& \dot{\mathbf{p}}_{\mathbf{i}}=\mathbf{v}_{\mathbf{i}} \\
& \dot{\mathbf{v}}_{\mathbf{i}}=\mathbf{u}_{\mathbf{i}}
\end{aligned}
$$

where $\mathbf{v}_{\mathbf{i}}$ and $\mathbf{u}_{\mathbf{i}}$ are the velocity and control input vector, respectively. In [8], control is based on the custom $\sigma$-norm which maps $\mathbb{R}^{n} \rightarrow \mathbb{R}^{+}$as:

$$
\forall \mathbf{z} \in \mathbb{R}^{n},\|\mathbf{z}\|_{\sigma}=\frac{1}{\epsilon}\left[\sqrt{1+\epsilon\|\mathbf{z}\|^{2}}-1\right]
$$

where $\epsilon>0$ and its gradient $\sigma_{\epsilon}(\mathbf{z})=\nabla\|\mathbf{z}\|_{\sigma}$ is written as:

$$
\sigma_{\epsilon}(\mathbf{z})=\frac{\mathbf{z}}{\sqrt{1+\epsilon\|\mathbf{z}\|^{2}}}=\frac{\mathbf{z}}{1+\epsilon\|\mathbf{z}\|_{\sigma}}
$$

Let $a_{i j}(\mathbf{p})$ be elements of a spatial adjacency matrix $\mathbf{A}(\mathbf{p})$, defined as follows:

$$
a_{i j}= \begin{cases}0 & \text { if } i=j \\ \rho_{h}\left(\left\|\mathbf{p}_{\mathbf{j}}-\mathbf{p}_{\mathbf{i}}\right\|_{\sigma} /\|c\|_{\sigma}\right) & \text { if } i \neq j\end{cases}
$$

where $\rho_{h}: \mathbb{R}^{+} \rightarrow[0,1]$ is a bump function with $h \in(0,1)$ :

$$
\rho_{h}(s)= \begin{cases}1, & s \in[0, h) \\ \frac{1}{2}\left[1+\cos \left(\pi \frac{(s-h)}{(1-h)}\right)\right], & s \in[h, 1] \\ 0, & \text { otherwise }\end{cases}
$$

The control law in [8] is then defined as follows:

$$
\begin{aligned}
\mathbf{u}_{\mathbf{i}} & =\sum_{j \in N_{i}^{\alpha}}\left[K_{p} \Phi_{\alpha}\left(\left\|\mathbf{p}_{\mathbf{j}}-\mathbf{p}_{\mathbf{i}}\right\|_{\sigma}\right) \mathbf{n}_{\mathbf{i j}}+K_{p}^{\prime} a_{i j}(\mathbf{p})\left(\mathbf{p}_{\mathbf{j}}-\mathbf{p}_{\mathbf{i}}\right)\right. \\
& \left.+K_{d} a_{i j}(\mathbf{p})\left(\mathbf{v}_{\mathbf{j}}-\mathbf{v}_{\mathbf{i}}\right)+K_{i} \int \Phi_{\alpha}\left(\left\|\mathbf{p}_{\mathbf{j}}-\mathbf{p}_{\mathbf{i}}\right\|_{\sigma}\right) \mathbf{n}_{\mathbf{i j}} d t\right] \\
& -c_{1}^{\gamma}\left(\mathbf{p}_{\mathbf{i}}-\mathbf{p}_{\mathbf{r}}\right)-c_{2}^{\gamma}\left(\mathbf{v}_{\mathbf{i}}-\mathbf{v}_{\mathbf{r}}\right)
\end{aligned}
$$

where:

- $K_{p}, K_{p}^{\prime}, K_{d}, K_{i}>0$ are consensus gains

- $\mathbf{n}_{\mathbf{i j}}=\sigma_{\epsilon}\left(\mathbf{p}_{\mathbf{j}}-\mathbf{p}_{\mathbf{i}}\right)$

- $\Phi_{\alpha}(s)=\frac{1}{2} \rho_{h}\left(s /\|c\|_{\sigma}\right)\left[(a+b) \sigma_{1}\left(s-\|d\|_{\sigma}+e\right)+(a-b)\right]$

- $\sigma_{1}(s)=s / \sqrt{1+s^{2}}$

- $0<a<b$ and $e=|a-b| / \sqrt{4 a b}$

- $p_{\mathbf{r}}$ and $\mathbf{v}_{\mathbf{r}}$ are the reference position and velocity, respectively

- $c_{1}^{\gamma}, c_{2}^{\gamma}>0$ are navigation gains, tuning the behavior with regards to position and velocity error, respectively.

This control law complies with the three rules of flocking defined in [6]. The inter-distances between robots are regulated by the first three terms, while the fourth and last term take care of velocity matching and navigation, respectively. 


\section{Obstacle avoidance for flocking}

Since the flocking algorithm proposed by [8] does not include any obstacle avoidance capability into the control law, we will now detail the obstacle avoidance algorithm from [9].

Fig. 2 shows the agent-based approach of representing obstacles. An $\alpha$-agent represents actual robots while a $\beta$ agent indicates the obstacles. The set of neighbors of node $i$ can be written as:

$$
\begin{aligned}
& N_{i}^{\alpha}=j \in \mathcal{V}_{\alpha}:\left\|\mathbf{p}_{\mathbf{j}}-\mathbf{p}_{\mathbf{i}}\right\|<c \\
& N_{i}^{\beta}=k \in \mathcal{V}_{\beta}:\left\|\hat{\mathbf{p}}_{\mathbf{i}, \mathbf{k}}-\mathbf{p}_{\mathbf{i}}\right\|<c^{\prime}
\end{aligned}
$$

The desired formation of the system, eq. (2), thus includes the agent-to-obstacle constraint as follows:

$$
\begin{cases}\left\|\mathbf{p}_{\mathbf{j}}-\mathbf{p}_{\mathbf{i}}\right\|=d & \forall j \in N_{i}^{\alpha} \\ \left\|\hat{\mathbf{p}}_{\mathbf{i}, \mathbf{k}}-\mathbf{p}_{\mathbf{i}}\right\|=d^{\prime} & \forall k \in N_{i}^{\beta}\end{cases}
$$

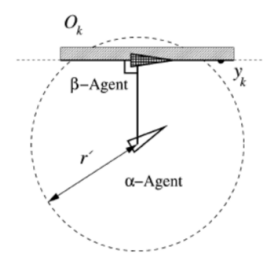

(a)

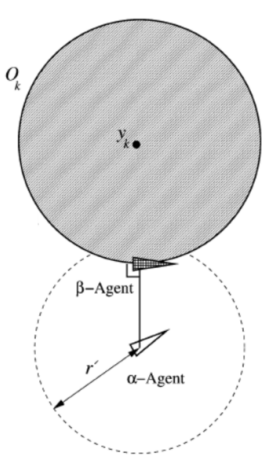

(b)
Fig. 2: Obstacles denoted by agent-based approach: (a) wall (b) spherical obstacles [9].

where $c^{\prime}$ and $d^{\prime}$ are the interaction range and inter-distance between $\alpha$-agent $i$ and $\beta$-agent $k$, respectively. $\hat{\mathbf{p}}_{\mathbf{i}, \mathbf{k}}$ is an estimated position, and $\hat{\mathbf{v}}_{\mathbf{i}, \mathbf{k}}$ and estimated velocity of the closest point from robot $i$ to obstacle $k$. Since obstacles are treated as virtual robots, the avoidance control law is similar to flocking control law based on a potential function, which is defined as following:

$$
\begin{aligned}
\mathbf{u}_{\mathbf{i}}^{\boldsymbol{\beta}} & =c_{1}^{\beta} \sum_{k \in N_{i}^{\beta}} \Phi_{\beta}\left(\left\|\hat{\mathbf{p}}_{\mathbf{i}, \mathbf{k}}-\mathbf{p}_{\mathbf{i}}\right\|_{\sigma}\right) \hat{\mathbf{n}}_{\mathbf{i}, \mathbf{k}} \\
& +c_{2}^{\beta} \sum_{k \in N_{i}^{\beta}} b_{i, k}(\mathbf{p})\left(\hat{\mathbf{v}}_{\mathbf{i}, \mathbf{k}}-\mathbf{v}_{\mathbf{i}}\right)
\end{aligned}
$$

where $c_{1}^{\beta}, c_{2}^{\beta}>0$ are gains, $\hat{\mathbf{n}}_{\mathbf{i}, \mathbf{k}}=\sigma_{\epsilon}\left(\hat{\mathbf{p}}_{\mathbf{i}, \mathbf{k}}-\mathbf{p}_{\mathbf{i}}\right)$, and $b_{i, k}(\mathbf{p})$, which is the heterogeneous adjacency between an $\alpha$-agent $i$ and its neighboring obstacle $k$ at $\hat{\mathbf{p}}_{\mathbf{i}, \mathbf{k}}$, is defined as:

$$
b_{i, k}(\mathbf{p})=\rho_{h}\left(\left\|\hat{\mathbf{p}}_{\mathbf{i}, \mathbf{k}}-\mathbf{p}_{\mathbf{i}}\right\|_{\sigma} / d_{\beta}\right)
$$

with $d_{\beta}=\left\|d^{\prime}\right\|_{\sigma}$ and the function defining the repulsive action can be expressed as follows:

$$
\Phi_{\beta}(s)=\rho_{h}\left(s / d_{\beta}\right)\left(\sigma_{1}\left(s-d_{\beta}\right)-1\right)
$$

Combining the control law (8) with the obstacle avoidance algorithm (11), the overall control law can be written as:

$$
\begin{aligned}
\mathbf{u}_{\mathbf{i}} & =\mathbf{u}_{\mathbf{i}}^{\boldsymbol{\alpha}}+\mathbf{u}_{\mathbf{i}}^{\boldsymbol{\beta}}+\mathbf{u}_{\mathbf{i}}^{\boldsymbol{\gamma}} \\
\mathbf{u}_{\mathbf{i}}^{\boldsymbol{\alpha}} & =\sum_{j \in N_{i}^{\alpha}}\left[K_{p} \Phi_{\alpha}\left(\left\|\mathbf{p}_{\mathbf{j}}-\mathbf{p}_{\mathbf{i}}\right\|_{\sigma}\right) \mathbf{n}_{\mathbf{i j}}+K_{p}^{\prime} a_{i j}(\mathbf{p})\left(\mathbf{p}_{\mathbf{j}}-\mathbf{p}_{\mathbf{i}}\right)\right. \\
& \left.+K_{d} a_{i j}(\mathbf{p})\left(\mathbf{v}_{\mathbf{j}}-\mathbf{v}_{\mathbf{i}}\right)+K_{i} \int \Phi_{\alpha}\left(\left\|\mathbf{p}_{\mathbf{j}}-\mathbf{p}_{\mathbf{i}}\right\|_{\sigma}\right) \mathbf{n}_{\mathbf{i j}} d t\right] \\
\mathbf{u}_{\mathbf{i}}^{\boldsymbol{\beta}} & =c_{1}^{\beta} \sum_{k \in N_{i}^{\beta}} \Phi_{\beta}\left(\left\|\hat{\mathbf{p}}_{\mathbf{i}, \mathbf{k}}-\mathbf{p}_{\mathbf{i}}\right\|_{\sigma}\right) \hat{\mathbf{n}}_{\mathbf{i}, \mathbf{k}} \\
& +c_{2}^{\beta} \sum_{k \in N_{i}^{\beta}} b_{i, k}(\mathbf{p})\left(\hat{\mathbf{v}}_{\mathbf{i}, \mathbf{k}}-\mathbf{v}_{\mathbf{i}}\right) \\
\mathbf{u}_{\mathbf{i}}^{\gamma} & =-c_{1}^{\gamma}\left(\mathbf{p}_{\mathbf{i}}-\mathbf{p}_{\mathbf{r}}\right)-c_{2}^{\gamma}\left(\mathbf{v}_{\mathbf{i}}-\mathbf{v}_{\mathbf{r}}\right)
\end{aligned}
$$

Equation (14) represents a complete control law for dynamic flocking. Each robot in the team will keep a certain distance from its neighbors, try to match each others' velocities, and avoids obstacles while navigating to a goal point.

\section{Formation CONTROL WITH OBSTACLE AVOIDANCE}

In this section, the model of a unicycle robot, and the use of static state feedback in order that the consensus approach can be applied on such robot is discussed. The proposed control algorithm for formation and obstacle avoidance is also introduced in this section.

\section{A. System model}

Consider a multi-robots system with $n$ unicycle robots, $\left(x_{i}, y_{i}\right)^{T} \in \mathbb{R}^{2}$ is the center of the $i$-th robot and $\theta_{i}, v_{i}$ and $\omega_{i}$ are its heading angle, linear velocity and angular velocity, respectively. The nonholonomic constraints of the wheeled mobile robot can be expressed as following:

$$
\left(\begin{array}{c}
\dot{x}_{i} \\
\dot{y}_{i} \\
\dot{\theta}_{i}
\end{array}\right)=\left(\begin{array}{cc}
\cos \theta_{i} & 0 \\
\sin \theta_{i} & 0 \\
0 & 1
\end{array}\right)\left(\begin{array}{c}
v_{i} \\
\omega_{i}
\end{array}\right)
$$

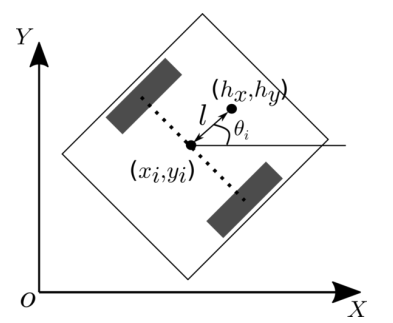

Fig. 3: A nonholonomic wheeled mobile robot.

We use a static feedback linearization method by defining an offset position from the robot center, as shown in Fig. 3. The relation of position between the offset point and the robot center can be written as follows [25]:

$$
\left(\begin{array}{l}
h_{x} \\
h_{y}
\end{array}\right)=\left(\begin{array}{l}
x_{i} \\
y_{i}
\end{array}\right)+l\left(\begin{array}{c}
\cos \theta_{i} \\
\sin \theta_{i}
\end{array}\right)
$$


Differentiating (16) with respect to time, the robot velocity can be found using following equation:

$$
\left(\begin{array}{c}
v_{i} \\
w_{i}
\end{array}\right)=\left(\begin{array}{cc}
\cos \theta_{i} & \sin \theta_{i} \\
-\frac{1}{l} \sin \theta_{i} & \frac{1}{l} \cos \theta_{i}
\end{array}\right)\left(\begin{array}{l}
\dot{h}_{x} \\
\dot{h}_{y}
\end{array}\right)
$$

Thus, the consensus control law can be applied directly to the offset position $\left(h_{x}, h_{y}\right)$ before using (17) to find the robot velocity with $l>0$.

\section{B. Control algorithm}

Our goal is to apply the control law in (14) to a single integrator system. Thus, the equation is modified to:

$$
\begin{aligned}
\mathbf{u}_{\mathbf{i}} & =\mathbf{u}_{\mathbf{i}}^{\boldsymbol{\alpha}}+\mathbf{u}_{\mathbf{i}}^{\boldsymbol{\beta}}+\mathbf{u}_{\mathbf{i}}^{\gamma} \\
\mathbf{u}_{\mathbf{i}}^{\boldsymbol{\alpha}} & =\sum_{j \in N_{i}^{\alpha}}\left[K_{p} \Phi_{\alpha}\left(\left\|\mathbf{p}_{\mathbf{j}}-\mathbf{p}_{\mathbf{i}}\right\|_{\sigma}\right) \mathbf{n}_{\mathbf{i j}}\right. \\
& \left.+K_{i} \int \Phi_{\alpha}\left(\left\|\mathbf{p}_{\mathbf{j}}-\mathbf{p}_{\mathbf{i}}\right\|_{\sigma}\right) \mathbf{n}_{\mathbf{i j}} d t\right] \\
\mathbf{u}_{\mathbf{i}}^{\boldsymbol{\beta}} & =c_{1}^{\beta} \sum_{k \in N_{i}^{\beta}} \Phi_{\beta}\left(\left\|\hat{\mathbf{p}}_{\mathbf{i}, \mathbf{k}}-\mathbf{p}_{\mathbf{i}}\right\|_{\sigma}\right) \hat{\mathbf{n}}_{\mathbf{i}, \mathbf{k}} \\
\mathbf{u}_{\mathbf{i}}^{\gamma} & =-\operatorname{sat}\left(c_{1}^{\gamma}\left(\mathbf{p}_{\mathbf{i}}-\mathbf{p}_{\mathbf{r}}\right)\right)
\end{aligned}
$$

where $\operatorname{sat}($.$) is a saturation function defined as:$

$$
\operatorname{sat}(x)=\left\{\begin{array}{lr}
x_{\text {min }}, & x \leq x_{\text {min }} \\
x, & x_{\text {min }}<x<x_{\text {max }} \\
x_{\text {max }}, & x \geq x_{\text {max }}
\end{array}\right.
$$

The value of $x_{\min }$ and $x_{\max }$ are chosen explicitly in order to ensure that the navigation term will not dominate over other control terms when the goal point is too far away. In most scenarios where the robots actually perform trajectory tracking, the goal point is in the vicinity of the robots and the saturation function may be eluded.

A simulation of (18) is shown in Fig. 4. It can be seen in Fig. $4 \mathrm{~b}$ that robot 0 (red) breaks formation in order to avoid the obstacle. More precisely, this robot avoids the obstacle from the left side while the other robots avoid it from the right side. This behavior is not desirable considering the conditions we have proposed in Section III, with formation keeping as a criterion.

In order to ensure the formation while navigating around the obstacle, we propose a new consensus obstacle avoidance scheme. In this approach, each robot will take into account its neighbors' obstacles as well. By doing so, it can anticipate incoming obstacles even they are not yet in its own detection range. Hence, the velocity of each robot will be synchronized while avoiding the obstacle. The obstacle avoidance term of (18), $\mathbf{u}_{\mathbf{i}}^{\boldsymbol{\beta}}$, now takes into account all potential obstacles for all robots:

$$
\begin{aligned}
\mathbf{u}_{\mathbf{i}}^{\boldsymbol{\beta}} & =c_{1}^{\beta}\left[\sum_{k \in N_{i}^{\beta}} \Phi_{\beta}\left(\left\|\hat{\mathbf{p}}_{\mathbf{i}, \mathbf{k}}-\mathbf{p}_{\mathbf{i}}\right\|_{\sigma}\right) \hat{\mathbf{n}}_{\mathbf{i}, \mathbf{k}}\right. \\
& \left.+\sum_{j \in N_{i}^{\alpha}} \sum_{k \in N_{j}^{\beta}} \Phi_{\beta}\left(\left\|\hat{\mathbf{p}}_{\mathbf{j}, \mathbf{k}}-\mathbf{p}_{\mathbf{j}}\right\|_{\sigma}\right) \hat{\mathbf{n}}_{\mathbf{j}, \mathbf{k}}\right]
\end{aligned}
$$

By defining as such, the fleet will react to the obstacles with the same motion preserving its shape. The simulation and experimental results can be found in the next section.

\section{RESULTS}

In this section, we validate the proposed control law in simulation as well as real experiment. In both simulation and experiment, ROS [26] is used as the middleware. In the case of simulation, Gazebo [27] is used to simulate four robots under one ROS master, and we simulate the control laws from (18) and (20). For experiment, we utilize four Huskys. The control law (20) is run in each Husky independently, only obstacle and its own positions, which are estimated using on-board sensors, in a known map are shared within a local network.

The value of parameters used in the simulation and experiment are shown in table I. The interaction range, $c$, for control law (18) is chosen according to the remark of authors in [9], while for (20), it is defined such that each robot becomes neighbor of all others in the team. The minimum and maximum values of the saturation function are -0.3 and 0.3 , respectively. A video of simulation and experimental result is available at this link ${ }^{1}$.

TABLE I: Value of parameters.

\begin{tabular}{|c|c||c|c|}
\hline Parameter & Value & Parameter & value $[\mathrm{m}]$ \\
\hline$a$ & 1.0 & $x_{c_{g}}$ & 8.0 \\
$b$ & 1.0 & $y_{c_{g}}$ & -3.0 \\
$h$ & 0.2 & $d$ & 2.0 \\
$\epsilon$ & 0.1 & $d^{\prime}$ & 1.5 \\
$K_{p}$ & 0.2 & $c$ for $(18)$ & 2.5 \\
$K_{i}$ & 0.05 & $c$ for $(20)$ & 5.0 \\
$c_{1}^{\beta}$ & 0.3 & $l$ & 0.2 \\
$c_{1}^{\gamma}$ & 0.25 & & \\
\hline
\end{tabular}

\section{A. Simulation}

The result of proposed control law (20) is shown in Fig. 5. In Fig. 5a, $d_{i j}$ represents the inter-distance between robots $i$ and $j$, where $i, j \in\{0,1,2,3\}$ and $i \neq j$. The inter-distances between each robot quickly converge and is stable over time. Moreover, the formation of the team is preserved even during obstacle avoidance motion. As shown in Fig. 5b, the shape is fixed throughout the navigation. This behavior is desirable, and we will see the actual implementation of this control algorithm in the next section.

The behavior is much better than in the naive approach shown in Fig. 4, using law (18). Indeed, In Fig. 4a it was clear that the inter-distances took a huge amount of time to stabilize. As already stated, it is clearly visible in Fig. 4b that one of the robots breaks off from the formation to avoid the obstacle. The formation shape is totally distorted, and restored only after the obstacle is passed through. This is, indeed, a common behavior as shown in [9].

${ }^{1}$ https://youtu.be/kS7HrOHnbho 


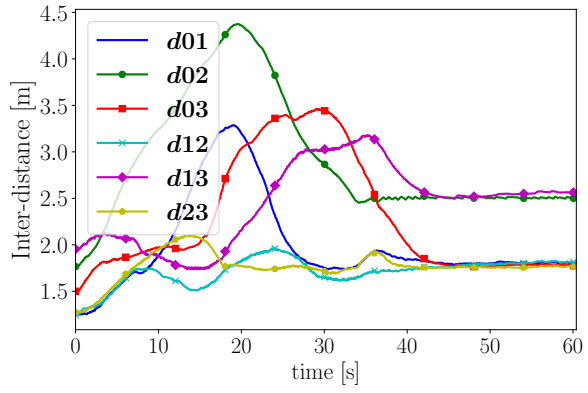

(a) Inter-distances between robots require huge amount of time to converge.

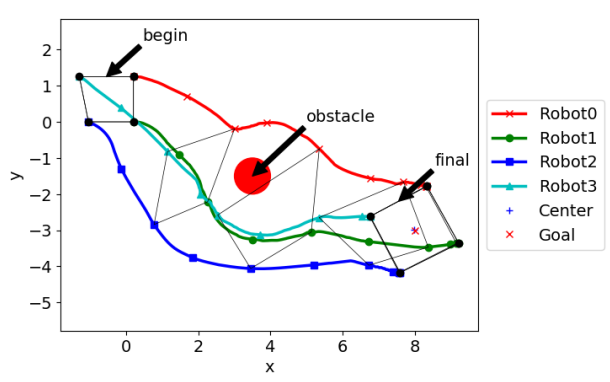

(b) Robots trajectories. Robot 0 breaks the formation in order to avoid the obstacle.

Fig. 4: Simulation of formation and obstacle avoidance using flocking / avoidance law (18).

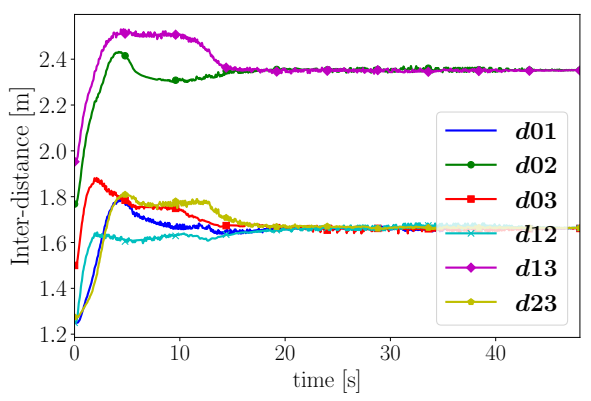

(a) Inter-distances between robots converge to the desired value from target formation.

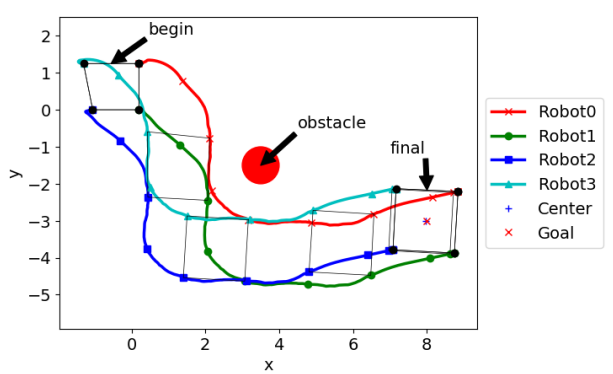

(b) Robots trajectories. Once formation is reached, it is kept while avoiding the obstacle.

Fig. 5: Simulation of formation and obstacle avoidance using proposed control law (20).

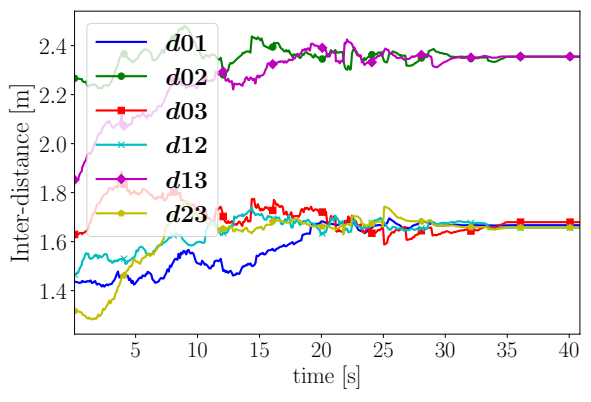

(a) Inter-distances between robots. Convergence to the desired value with oscillations due to the low-level controller.

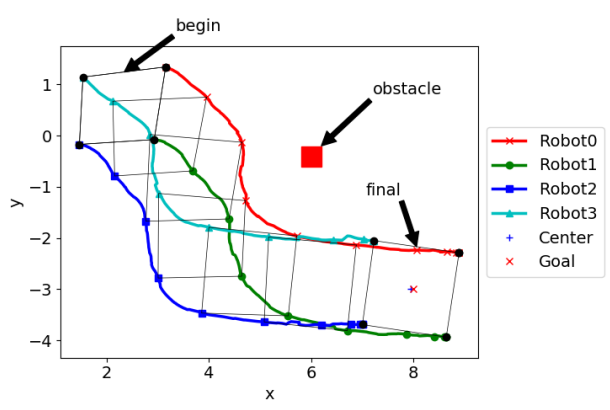

(b) Robots trajectories. Once formation is reached, it is kept while avoiding the obstacle.

Fig. 6: Real experiment of formation and obstacle avoidance using proposed control law (20).
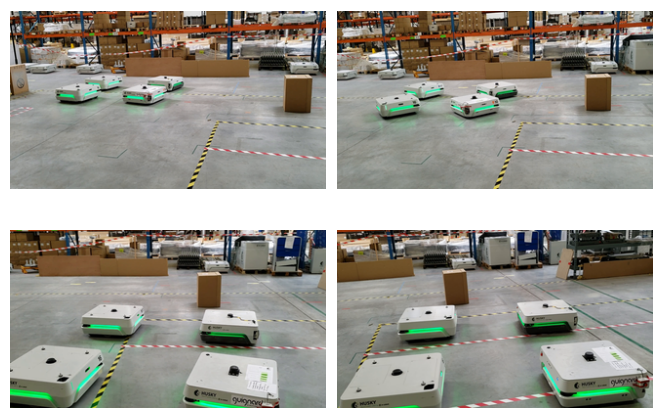
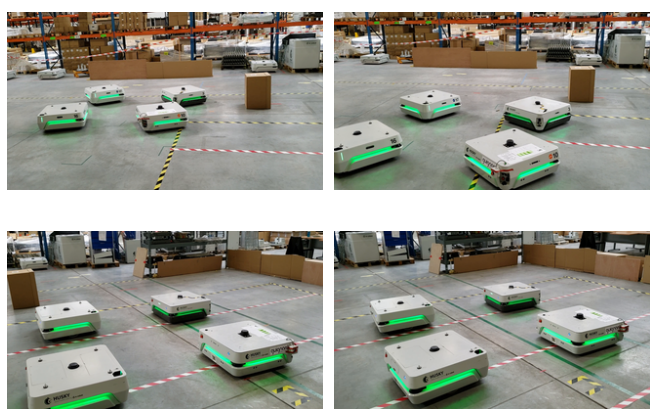

Fig. 7: Snapshots of the experiment. The fleet navigation from one point to another, and its formation is kept while avoiding the obstacle that is not already known in the map. 


\section{B. Experiment}

The proposed control law is now applied on four real Husky platforms for experimental validation, and the result is shown in Fig. 6. From Fig. 6a, we can notice the convergence of the inter-distances between robot even though the oscillation is a bit higher than in simulation, which is due to the current low-level control of Husky. Fig. 6b shows the trajectories of the four Huskys. As in simulation, the formation does not break while the team maneuvers away from the obstacle. Thus, all conditions we defined in section III are achieved by using the proposed control law.

Fig. 7 shows a sequence of snapshots of this experiment, where the fleet has to move from one point to another. An obstacle (carton box) was placed in between the two points. This obstacle is not already known in the map, hence no initial path planning can try to avoid it: it has to be detected and avoided by the whole fleet during the navigation.

\section{CONCLUSIONS}

In this paper, we have revised existing flocking algorithms such that it can be applied in nonholonomic robot system. A combined consensus control for formation and obstacle avoidance is proposed, and it is able to do formation and navigation of the fleet's barycenter to a goal point without any collision with the obstacles. The validation of this control law shows its effectiveness in simulations as well as real experiment using actual industrial robots, Huskys.

Future work will focus on the main limitations of the proposed approach, Our future work will focus on improving the existing algorithm such that the inter-distances of all side neighbors converge to the desired value when robots in diagonal are neighbors, which is not the case for current algorithm. Moreover, since wireless communication is always more preferable in large industrial environment, transmission delay between robots is inevitable. This problem will be also addressed in the future study.

\section{REFERENCES}

[1] S. Yu and J. C. Barca, Autonomous formation selection for ground moving multi-robot systems, IEEE Int. Conf. on Advanced Intelligent Mechatronics, Busan, Korea, Jul., 2015

[2] A. Gautam and S. Mohan, A Review of Research in Multi-Robot Systems, IEEE Int. Conf. on Industrial and Information System, Chennai, India, Aug., 2012

[3] M. Popescu, H. Rivano, and O. Simonin, Multi-cycle Coverage for Multi-robot Patrolling - application to data collection in WSNs, Journées Francophones sur la Planification, la Décision et l'Apprentissage pour la conduite de systèmes, Caen, France, Jul., 2017

[4] A. Birk and S. Carpin, Merging Occupancy Grid Maps From Multiple Robots, in Proc. of the IEEE, vol. 94, pp. 1384-1397, Jul., 2006

[5] Z. Miao, Y. H. Liu, Y. Wang, G. Yi, and R. Fierro, Distributed estimation and control for leader-follwing formations of nonholonomic mobile robots, IEEE Trans. on Automation Science and Engineering, vol. 15, pp. 1946-1954, Oct. 2018

[6] C. W. Reynolds, Flocks, herds, and schools: a distributed behavioral model, Computer Graphics, vol. 21, pp. 25-34, Jul., 1987

[7] D. V. Dimarogonas and K. J. Kyriakopoulos, A connection between formation control and flocking behavior in nonholonomic multiagent systems, IEEE Int. Conf. on Robotics and Automation, Florida, USA, May, 2006

[8] O. Saif, I. Fantoni, and A. Zavala-Rio, Distributed integral control of multiple UAVs: precise flocking and navigation, IET Control Theory \& Applications, vol. 13, pp. 2008-2017, Aug., 2019
[9] R. Olfati-Saber, Flocking for multi-agent dynamic systems: algorithms and theory, IEEE Trans. on Automatic Control, vol. 51, Mar., 2006

[10] Z. Hou, W. Wang, G. Zhang, and C. Han, A survey on the formation control of multiple quadrotors, 14th Int. Conf. on Ubiquitous Robots and Ambient Intelligence (URAI), Jeju, Korea, Jun., 2017

[11] A. Bautista-Castillo, C. López-Franco, and Emmanuel Nuño, Consensus-based formation control for multiple nonholonomic robots, IEEE Int. Autumn Meeting on Power, Electronics and Computing, Ixtapa, Mexico, Nov., 2016

[12] M. C. Fan, Adaptive consensus of multi-agent systems with unknown control coefficients and input saturation, 29th Chinese Control and Decision Conf., Chongqing, China, May, 2017

[13] S. I. Han, Prescribed consensus and formation error constrained finitetime sliding mode control for multi-agent mobile robot systems, IET Control Theory \& Applications, vol. 12, pp. 282-290, Jan., 2018

[14] X. Xu, L. Liu, and G. Feng, Consensus of single integrator multi-agent systems with unbounded transmission delays, 13th IEEE Int. Conf. on Control \& Automation, Ohrid, Macedonia, Jul., 2017

[15] Z. Peng, G. Wen, A. Rahmani, and Y. Yu, Distributed consensus-based formation control for multiple nonholonomic mobile robots with a specified reference trajectory, Int. Journal of Systems Science, vol. 46, pp. 1447-1457, Jun., 2015

[16] D. Geng, Q. Wu, and C. Du, Consensus tracking of nonlinear secondorder multi-agent systems with external disturbance, 4th Int. Conf. on Info. Science and Control Engineering, Changsha, China, Jul., 2017

[17] B. Lei and W. Li, Formation control for multi-robots based on flocking algorithm, Int. Conf. on Intelligent Robotics and Applications, Wuhan, China, Oct. 2008

[18] H. Cao, J. Chen, Y. Mao, H. Fang, and H. Liu, Formation control based on flocking algorithm in multi-agent system, World Congress on Intelligent Control and Automation, Jinan, China, Jul., 2010

[19] D. Sakai, H. Fukushima, and F. Matsuno, Flocking for multirobots without distinguishing robots and obstacles, IEEE Trans. on Control Systems Technology, vol. 25, pp. 1019-2027 May, 2017

[20] S. Fuady, A. R. Ibrahim, and R. T. Bambang, Distributed formation control of multi-robot system with obstacle avoidance, Int. Conf. on Robotics, Biominetics, Intelligent Computational Systems, Yogyakarta, Indonesia, Nov., 2013

[21] R. Toyota and T. Namerikawa, Formation control of multi-agent system considering obstacle avoidance, SICE Annual Conf., Kanazawa, Japan, Sep., 2017

[22] E-COBOT, HUSKY, 2019. [Online]. Available: http://http://cobothusky.com/. [Accessed Aug. 23, 2019]

[23] R. Diestel, Graph theory, Grad. Texts in Mathematics, vol. 173, 2005

[24] W. Ren, R. W. Beard, and E. M. Atkins, Information consensus in multivehicle cooperative control, IEEE Control Systems Magazine, vol. 27, pp. 71-82, Apr., 2007

[25] W. Ren and R. W. Beard, Distributed consensus in multi-vehicle cooperative control: theory and applications, Springer-Verlag London, pp. 198-199, 2008

[26] Open Source Robotics Foundation, ROS, 2019. [Online]. Available: http://ros.org/. [Accessed Aug. 23, 2019]

[27] Open Source Robotics Foundation, Gazebo, 2019. [Online]. Available: http://gazebosim.org/. [Accessed Aug. 23, 2019] 